Highlighted Research Paper: Chemogenetic Suppression of Medial Prefrontal-Dorsal Hippocampal Interactions Prevents Estrogenic Enhancement of Memory Consolidation in Female Mice by, Jennifer J. Tuscher, Lisa R. Taxier, Jayson C. Schalk, Jacqueline M. Haertel, and Karyn M. Frick.

\title{
Concurrent Medial Prefrontal Cortex and Dorsal Hippocampal Activity Is Required for Estradiol- Mediated Effects on Object Memory and Spatial Memory Consolidation
}

\author{
Rosalind S.E. Carney, DPhil
}

Steroid hormones such as estrogens and androgens affect the nervous system during in utero development and throughout adolescence, adulthood, and aging (Choleris et al., 2018). Steroid hormones have been associated with a prevalence in either men or women of certain neurologic disorders. For instance, Alzheimer's disease and schizophrenia affect more women than men and vice versa, respectively (Irvine et al., 2012; Jackson et al., 2013). Studies have also shown that $17 \beta$-estradiol $\left(E_{2}\right)$, the predominant and most potent form of estrogen, can alter hippocampal neuroplasticity and therefore affect learning and memory. Exogenous $\mathrm{E}_{2}$ administration into the dorsal hippocampus (DH) of young female rodents enhances memory consolidation as shown by object recognition (OR) and object placement (OP; also known as object location) tasks (Tuscher et al., 2015). OR and OP are two object-based one-trial learning tasks that capitalize on rodents' innate interest in novelty and relate to the "what" and "where" aspects of episodic memory consolidation and spatial memory, respectively (Frick et al., 2018a). DH $E_{2}$ infusion immediately after the OR/OP training period restricts the effects of $E_{2}$ to the time frame of memory consolidation (Tuscher et al., 2015). Post-training DH $\mathrm{E}_{2}$ infusion leads to an increase in spine density of CA1 pyramidal neurons during the time frame in which memory consolidation occurs (Gould et al., 1990; Woolley et al., 1990; Woolley and McEwen, 1993; MacLusky et al., 2005; Phan et al., 2012). Increased dendritic spine density in response to post-training $\mathrm{E}_{2} \mathrm{DH}$ infusion was also observed in the medial prefrontal cortex (mPFC; Tuscher

Received July 12, 2019; accepted July 12, 2019; First published August 20, 2019.

https://doi.org/10.1523/ENEURO.0271-19.2019

This is an open-access article distributed under the terms of the Creative Commons Attribution 4.0 International license, which permits unrestricted use, distribution and reproduction in any medium provided that the original work is properly attributed. et al., 2016), a structure that expresses estrogen receptors and is also involved in memory consolidation. This observation prompted Tuscher and colleagues to examine in their eNeuro publication the effects of local infusion of $E_{2}$ into the $\mathrm{mPFC}$ and the requirement for concurrent activity of the mPFC in $\mathrm{E}_{2}$-mediated memory consolidation in the $\mathrm{DH}$.

Young female mice were ovariectomized (OVX) to eliminate the ovarian source of $E_{2}$ that fluctuates during the estrous cycle, the rodent version of the menstrual cycle (Tuscher et al., 2015). Figure 1 shows a schematic illustration of the experimental approach. For OR training, a mouse was placed in a white square arena in which the upper left and right corners contained identical objects, one in each corner (Fig. 1A). The time spent exploring each object was recorded using tracking software, and mice remained in the arena until they had accumulated 30 $s$ exploring the objects. If the mice remembered the identity of the training objects during testing $48 \mathrm{~h}$ later, then they should spend more time than chance $(>15 \mathrm{~s}$ ) exploring a novel object that had replaced one of the original training objects in the upper corners of the arena (Fig. 1A). The OP assessment differed from that of OR in two aspects: (1) OP testing occurred $24 \mathrm{~h}$ after training; and (2) whereas one of the training objects encountered during training remained in its original location during testing, the second training object had been moved to a lower corner of the arena (Fig. 1B). Spending more time than chance with the moved object indicated that spatial memory was intact.

OVX mice were implanted with bilateral cannulae targeted to the mPFC one week before training in the OR and OP tasks, which were performed in separate groups of mice. Immediately after training, mice received mPFC infusions of either cyclodextrin-encapsulated $\mathrm{E}_{2}$ dissolved in sterile saline or the vehicle control, 2-hydroxypropyl- $\beta$ cyclodextrin (HBC) dissolved to the same concentration 


\section{A Object Recognition}

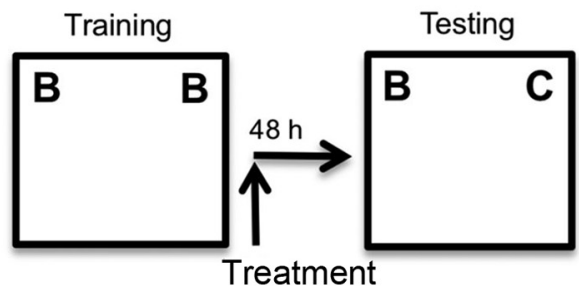

\section{B Object Placement}

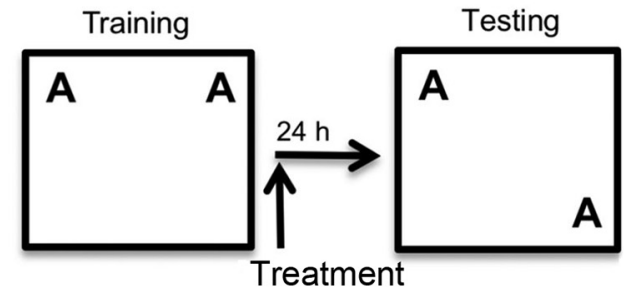

Figure 1. Schematic illustration of OR and OP behavioral assessments. $\boldsymbol{A}$, Treatment administered after training reveals treatment effect on memory consolidation of a novel object versus a familiar object. $\boldsymbol{B}$, Treatment administered after training reveals treatment effect on memory consolidation of the placement of a previously encountered object in a new location versus original placement of another previously encountered object (Adapted from Figure 1 in Tuscher et al., 2019).

of cyclodextrin as the $\mathrm{E}_{2}$-containing solution. In both the $\mathrm{OR}$ and $\mathrm{OP}$ behavioral assessments, $\mathrm{E}_{2}$-infused mice spent more time exploring the novel or moved objects than would be expected by chance alone and significantly more time compared to vehicle-infused mice (Fig. 2). These observations showed that local effects of $E_{2}$ within the mPFC replicate the enhancement of memory consolidation and spatial memory that resulted from local infusion of $\mathrm{E}_{2}$ within the $\mathrm{DH}$.

Although a causal link between $\mathrm{E}_{2}$-mediated spinogenesis and memory consolidation has not yet been established (Sheppard et al., 2019), signaling mechanisms are common to both processes (Frick et al., 2018a). Therefore, Tuscher and colleagues examined local $E_{2}$-mediated effects on spinogenesis in the mPFC. Two weeks after the completion of the OR and OP behavioral assessments, mice received an infusion of $E_{2}$ or vehicle control into the $\mathrm{mPFC} ; 2 \mathrm{~h}$ later the brains of the mice were harvested and processed for Golgi staining. Dendritic spine analysis was performed in regions in which increased $\mathrm{E}_{2}$-mediated spinogenesis was previously observed, namely, Layer II/III of the mPFC and CA1 of the DH. Spine density was increased apically but not basically when $E_{2}$ was locally infused into the mPFC, but spine density in the $\mathrm{DH}$ was unchanged. It is not known why local infusion of $\mathrm{E}_{2}$ did not affect spinogenesis in the $\mathrm{DH}$; perhaps the time frame was insufficient to detect any alterations or $E_{2}$ infusion directly into the $\mathrm{DH}$ has more hierarchical, rather than reciprocal, effects on another brain region.

The observations described thus far provided evidence that $E_{2}$ acting locally in the mPFC enhances memory consolidation and increases local spinogenesis. Next, the authors sought to determine if concurrent activation of the mPFC is required for enhanced $E_{2}$-mediated, DH-dependent memory consolidation. To do so, they employed a designer receptors exclusively activated by designer drugs (DREADD) approach to suppress activity in the mPFC while simultaneously infusing $\mathrm{E}_{2}$ into the $\mathrm{DH}$. Immediately after ovariectomy, viral DREADD constructs or saline (sham condition) were infused bilaterally into the mPFC, and cannulae were also placed bilaterally in the $\mathrm{DH}$ for future infusions. The AAV-CaMKII $\alpha$-eGFP construct induced expression of green fluorescent protein (GFP) in excitatory neurons in the MPFC three weeks later, at the time when behavioral assessments were initiated. At the time of the surgical procedure, another group of OVX mice received bilateral mPFC injections of an AAV-CaMKII $\alpha$ HA-hM4Di-IRES-mCitrine viral construct. Binding of the
A

48 h Object Recognition

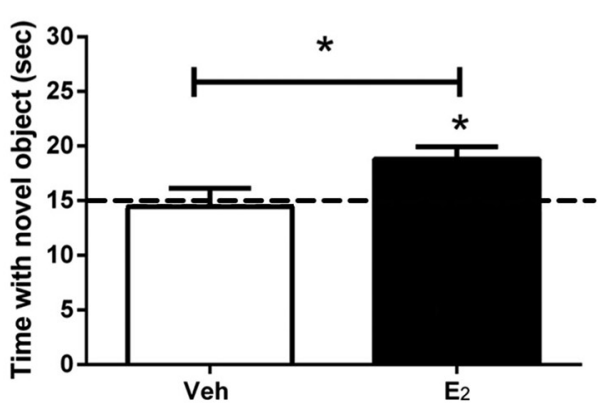

B

24 h Object Placement

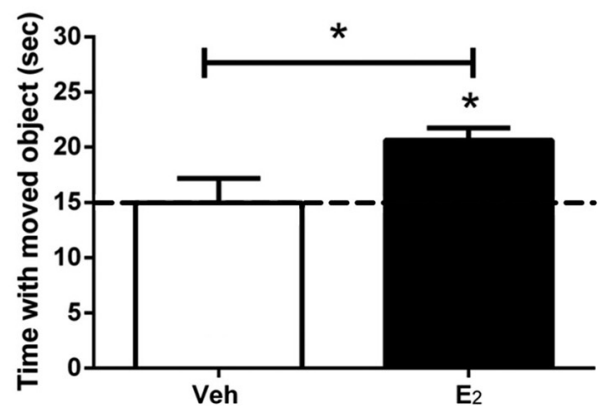

Figure 2. Infusion of $E_{2}$ into the mPFC immediately after training enhances memory consolidation. Mice infused with $E_{2}$ directly into the mPFC spent significantly more time than chance (dashed line at $15 \mathrm{~s}$ ) with the novel object $(\boldsymbol{A})$ when tested $48 \mathrm{~h}$ after training or with the moved object $(\boldsymbol{B}) 24 \mathrm{~h}$ after training. Mice infused with vehicle (Veh) into the mPFC did not spend more time than chance with the novel $(\boldsymbol{A})$ or moved $(\boldsymbol{B})$ objects. These data suggest that $\mathrm{E}_{2}$ can improve the consolidation of object memories by acting directly within the $\mathrm{mPFC}$. Bars represent the mean $\pm \mathrm{SEM} ; * p<0.05$ relative to chance and the vehicle group (Adapted from Figure 1 in Tuscher et al., 2019). 
A

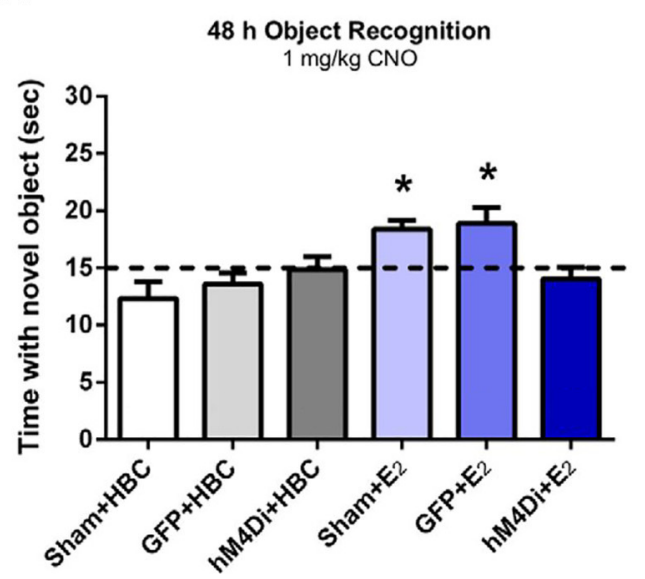

B

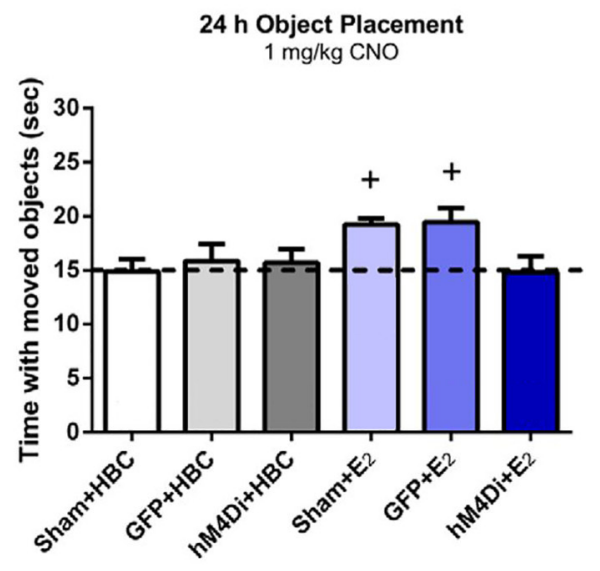

Figure 3. Chemogenetic suppression of the mPFC immediately after training prevents the memory enhancement induced by $\mathrm{DH} \mathrm{E}_{2}$ infusion. Sham, GFP, or hM4Di control groups receiving CNO + Veh did not spend significantly more time than chance (15 s) with the novel object $(\boldsymbol{A})$ when tested $48 \mathrm{~h}$ after training or with the moved object $(\boldsymbol{B}) 24 \mathrm{~h}$ after training. In contrast, sham or GFP mice receiving $\mathrm{CNO}+\mathrm{E}_{2}$ immediately after training did spend significantly more time than chance with the novel $(\boldsymbol{A})$ and moved $(\boldsymbol{B})$ and objects, displaying intact $\mathrm{OR}$ and $\mathrm{OP}$ memory. However, hM4Di mice treated with $\mathrm{CNO}+\mathrm{E}_{2}$ immediately after training did not demonstrate intact memory, suggesting that DREADD-mediated suppression of the mPFC blocks the beneficial mnemonic effects of $\mathrm{DH}$-infused $\mathrm{E}_{2}$. Bars represent the mean $\pm \mathrm{SEM} ; * p<0.05$ relative to chance, all Veh-infused groups, and the hM4Di- $\mathrm{E}_{2}$ group; $+p$ $<0.05$ relative to chance and the sham + Veh-infused and hM4Di- $E_{2}$ groups (Adapted from Figure 4 in Tuscher et al., 2019 ).

clozapine-N-oxide (CNO) ligand to the hM4Di DREADD suppresses the activity of the excitatory CaMKII $\alpha$ expressing cells, thereby inhibiting the mPFC. After training in the OR or OP tasks, mice received $\mathrm{DH}$ infusion of $\mathrm{E}_{2}$ or vehicle control at the same time that $\mathrm{CNO}$ was administered systemically to all experimental groups, at a concentration of CNO that does not impair memory consolidation on its own (Tuscher et al., 2018). In both the OR and OP tasks, memory consolidation was enhanced in mice in which $E_{2}$ was infused directly into the $\mathrm{DH}$ but in which mPFC activity was not suppressed (sham $+E_{2}$, GFP $+E_{2}$ groups; Fig. 3) compared to experimental groups that received vehicle control (sham + HBC, GFP + HBC, hM4Di + HBC; Fig. 3). Suppression of excitatory signaling in the MPFC blocked the $\mathrm{E}_{2}$-mediated $\mathrm{DH}$-dependent enhancement of memory consolidation (hM4Di + HBC; Fig. 3) showing, for the first time, that $\mathrm{mPFC}$ activity is required for memory consolidation when $E_{2}$ is locally infused into the $\mathrm{DH}$.

The eNeuro publication by Tuscher and colleagues highlights the complexity of $\mathrm{E}_{2}$-mediated memory consolidation processes and raises several new avenues of research. The finding that local $E_{2}$ infusion into the mPFC affects a cognitive process that is heavily associated with the hippocampus underscores the importance of research into other brain structures involved in cognition that express steroid receptors. The mPFC and DH interaction could be regulated by direct or indirect neural circuitry. It will also be interesting to determine if local effects of $E_{2}$ within the mPFC involve classic, genomic signaling via estrogen receptors $\mathrm{ER} \alpha$ and $\mathrm{ER} B$ or the rapid signaling that results from extracellular ligand binding to the G protein-coupled receptor GPER. Coupling the use of estrogen agonists and antagonists in the $\mathrm{DH}$ with chemogenetic suppression of other brain regions will help resolve the interactions between signaling mechanisms and neural circuitry. Such receptor manipulation could also reveal the relevance of local estradiol production within the hippocampus that is unaffected by ovariectomy.

Tuscher and colleagues' findings in this eNeuro publication may also help resolve discrepancies in the literature regarding the effects of $E_{2}$ on cognition by emphasizing the need to examine other brain regions involved in memory consolidation. But more complexities exist, such as the effects of steroid signaling in young versus older or female versus male rodent models (Frick et al., 2018b). It was expected that hormone replacement therapy in postmenopausal women would have a protective effect against cognitive dysfunction. However, some of these studies used conjugated equine estrogens, rather than $E_{2}$, which may explain the lack of beneficial effects (Luine, 2014). Moreover, it has recently been shown in a mouse model of Alzheimer's disease that $E_{2}$ fluctuations during the ovarian cycle have adverse effects on cognition (Broestl et al., 2018). Therefore, a plethora of age, sex, and brain region considerations may be needed to fully elucidate new targets for drug development that address steroid-related effects on cognitive functions, such as memory consolidation.

\section{References}

Broestl L, Worden K, Moreno AJ, Davis EJ, Wang D, Garay B, Singh T, Verret L, Palop JJ, Dubal DB (2018) Ovarian cycle stages modulate Alzheimer-related cognitive and brain network alterations in female mice. eNeuro 5: ENEURO.0132-17.2018.

Choleris E, Galea LAM, Sohrabji F, Frick KM (2018) Sex differences in the brain: implications for behavioral and biomedical research. Neurosci Biobehav Rev 85:126-145. 
Frick KM, Tuscher JJ, Koss WA, Kim J, Taxier LR (2018a) Estrogenic regulation of memory consolidation: a look beyond the hippocampus, ovaries, and females. Physiol Behav 187:57-66.

Frick KM, Kim J, Koss WA (2018b) Estradiol and hippocampal memory in female and male rodents. Curr Opin Behav Sci 23:65-74.

Gould E, Woolley CS, Frankfurt M, McEwen BS (1990) Gonadal steroids regulate dendritic spine density in hippocampal pyramidal cells in adulthood. J Neurosci 10:1286-1291.

Irvine K, Laws KR, Gale TM, Kondel TK (2012) Greater cognitive deterioration in women than men with Alzheimer's disease: a meta analysis. J Clin Exp Neuropsychol 34:989-998.

Jackson D, Kirkbride J, Croudace T, Morgan C, Boydell J, Errazuriz A, Murray RM, Jones PB (2013) Meta-analytic approaches to determine gender differences in the age-incidence characteristics of schizophrenia and related psychoses. Int $\mathrm{J}$ Methods Psychiatr Res 22:36-45.

Luine VN (2014) Estradiol and cognitive function: past, present and future. Horm Behav 66:602-618.

MacLusky NJ, Luine VN, Hajszan T, Leranth C (2005) The 17alpha and 17 beta isomers of estradiol both induce rapid spine synapse formation in the CA1 hippocampal subfield of ovariectomized female rats. Endocrinology 146:287-293.

Phan A, Gabor CS, Favaro KJ, Kaschack S, Armstrong JN, MacLusky NJ, Choleris E (2012) Low doses of $17 \beta$-estradiol rapidly improve learning and increase hippocampal dendritic spines. Neuropsychopharmacology 37:2299-2309.
Sheppard PAS, Choleris E, Galea L (2019) Structural plasticity of the hippocampus in response to estrogens in female rodents. Mol Brain 12:22.

Tuscher JJ, Fortress AM, Kim J, Frick KM (2015) Regulation of object recognition and object placement by ovarian sex steroid hormones. Behav Brain Res 285:140-157.

Tuscher JJ, Luine V, Frankfurt M, Frick KM (2016) Estradiol-mediated spine changes in the dorsal hippocampus and medial prefrontal cortex of ovariectomized female mice depend on ERK and mTOR activation in the dorsal hippocampus. J Neurosci 36:1483-1489.

Tuscher JJ, Taxier LR, Fortress AM, Frick KM (2018) Chemogenetic inactivation of the dorsal hippocampus and medial prefrontal cortex, individually and concurrently, impairs object recognition and spatial memory consolidation in female mice. Neurobiol Learn Mem 156:103-116.

Tuscher JJ, Taxier LR, Schalk JC, Haertel JM, Frick KM (2019) Chemogenetic suppression of medial prefrontal-dorsal hippocampal interactions prevents estrogenic enhancement of memory consolidation in female mice. eNeuro 6: ENEURO.0451-18.2019

Woolley CS, McEwen BS (1993) Roles of estradiol and progesterone in regulation of hippocampal dendritic spine density during the estrous cycle in the rat. J Comp Neurol 336:293-306.

Woolley CS, Gould E, Frankfurt M, McEwen BS (1990) Naturally occurring fluctuation in dendritic spine density on adult hippocampal pyramidal neurons. J Neurosci 10:4035-4039. 